\title{
Kidney Organoids Generated from iPS Cells Obtained from the Erythroid Progenitors of Patients with Autosomal Dominant Polycystic Kidney Disease
}

\section{Roberta Facioli ( $\sim$ facioli.roberta@unifesp.br)}

Universidade Federal de Sao Paulo - Campus Guarulhos https://orcid.org/0000-0002-7835-5843

Fernando Lojudice

Universidade de Sao Paulo

Ana Anauate

Universidade Federal de Sao Paulo

Edgar Maquigussa

Universidade Federal de Sao Paulo

José Luiz Nishiura

Universidade Federal de Sao Paulo

Ita Heilberg

Universidade Federal de Sao Paulo

Maricleide Sogayar

Universidade de Sao Paulo

Mirian Boim

Universidade Federal de Sao Paulo

\section{Research}

Keywords: Autosomal Dominant Polycystic Kidney Disease (ADPKD), induced pluripotent stem cells (iPSCs), erythroid progenitors, renal organoids

Posted Date: August 2nd, 2020

DOl: https://doi.org/10.21203/rs.3.rs-49228/v1

License: (9) (1) This work is licensed under a Creative Commons Attribution 4.0 International License. Read Full License 


\section{Abstract}

Background. Kidney organoids have been broadly obtained from commercially available induced pluripotent stem cells (iPSCs); however, it has been a great challenge to efficiently produce renal organoid models from patients with autosomal dominant polycystic kidney disease (ADPKD) that recapitulate both embryogenesis and the mechanisms of cystogenesis.

Methods. Blood erythroid progenitors (EPs) from two ADPKD patients and one healthy control (HC) donor were reprogrammed by an episomal vector into iPSCs, which were differentiated into renal tubular organoids and then stimulated by forskolin to induce cysts formation.

Results. iPSCs derived from EPs exhibited all characteristics of pluripotency and were able to differentiate into all three germ layers. 3D tubular organoids were generated from single cells after 28 days in Matrigel. $\mathrm{HC}$ and ADPKD organoids did not spontaneously form cysts, but upon forskolin stimulation, cysts-like structures were observed in the ADPKD organoids but not in the HC-derived organoids.

Conclusion. The findings of this study showed that kidney organoids were successfully generated from the blood EP cells of ADPKD patients and a healthy control donor. This approach should contribute as a powerful tool for embryonic kidney developing model which is able to recapitulate the very early pathophysiological mechanisms involved in cytogenesis.

\section{Introduction}

Autosomal dominant polycystic kidney disease (ADPKD) is the most frequent genetic cause of chronic kidney disease (CKD) worldwide, and it is one of the most prevalent inherited monogenic disorders, affecting a population estimated at 1:400-1000 ${ }^{1}$. ADPKD is caused by mutations in the PKD1 and PKD2 genes, coding for polycystin-1 (PC1) and polycystin-2 (PC2), respectively, resulting in cilia alterations and cyst formation. Both proteins modulate numerous molecular pathways as well as tissue morphogenesis and function. However, the exact molecular mechanisms underlying cystogenesis remain unclear. It has been well accepted that a germline mutation followed by a somatic mutation (second-hit) in the normal allele is necessary for cystogenesis ${ }^{2,3}$. Moreover, based on results from orthologous mouse models, it has been demonstrated that an additional renal insult (third-hit) is required for rapid and severe cyst growth in mature kidneys ${ }^{4-6}$. It seems that in humans, most second hits occur during renal development, leading to cystic formation and growth even in utero. Therefore, the severity and the rate of progression of kidney disease resulting from PKD1 and PKD2 mutations may be influenced by several factors, including the timing of inactivation of the genes, kidney developmental stage, environmental influence, presence of concomitant renal lesions, and diversity of genetic mutations.

Although there has been innumerable data concerning the pathophysiological mechanisms of ADPKD collected from studies employing orthologous and nonorthologous mouse models, only a handful of 
studies focusing on human cellular models of cystogenesis are available 7 . In 2007, Takahashi et al ${ }^{8}$ successfully reprogrammed somatic cells into pluripotent stem cells with embryonic properties, naming them "induced pluripotent stem cells" (iPSCs). Since then, many somatic cell sources have been reprogrammed into iPSCs from either animal or human tissues (hiPSCs) ${ }^{8}$, with adult fibroblasts representing the main source of the latter. In 2013, Freedman et al ${ }^{9}$ induced hiPSCs from the fibroblasts of ADPKD patients and found reduced ciliary expression of polycystin-2, supporting the use of such cells to investigate polycystic disease (PKD) pathophysiology. More recently, Chen et al reported in 2016 that they had obtained hiPS cells from erythroid progenitor cells ${ }^{10}$.

Over the last few years, there have been significant advances in the generation of 3-dimensional organoids from iPSCs ${ }^{8,11-13}$. Primary kidney tubular epithelial organoids can be derived from kidney tissue, as well as from urine, and these organoids are an important tool for personalized disease modeling, as shown by Schutgens et al ${ }^{14}$. In subsequent studies, Freedman et al ${ }^{15}$ were able to generate kidney organoids derived from a commercially available hiPS cell line, establishing a protocol to generate the cell types present in the metanephric mass that give rise to all structures of the nephron. Then, by knocking down the PKD1 or PKD2 genes, these investigators observed cyst formation from kidney tubules. In view of all these advanced and innovative technologies, we aimed to obtain iPSCs from the circulating erythroid progenitor cells of ADPKD patients and test the development of kidney organoids in vitro to recapitulate the steps involved in organogenesis, trying to evoke some pathophysiological events related to early cystogenesis.

\section{Methods}

Blood samples were collected from two unrelated female patients (PT1 and PT2) clinically diagnosed with ADPKD (PT1: 66 years old, eGFR $43.8 \mathrm{~mL} / \mathrm{min}$ per $1.73 \mathrm{~m} 2$ and PT2: 56 years old, eGFR $60.2 \mathrm{~mL} / \mathrm{min}$ per $1.73 \mathrm{~m} 2$ ), followed up at the Polycystic Kidney Disease Outpatient Clinic of the Nephrology Division of the Federal University of São Paulo (UNIFESP), and another sample was collected from one healthy female, which served as a control. The diagnosis of ADPKD was based on a positive family history and renal cysts according to the ultrasonographic diagnostic criteria by Pei et $\mathrm{al}^{16}$ (family pedigrees are presented in supplementary Figure S1A). Both patients had cystic kidneys and livers (Magnetic Resonance Images are presented in supplementary Figure S1B). The study was reviewed and approved by the Ethics Advisory Committee of the University (Nr.1199.-0079-10/2018). After an interview to explain the purpose of the study, both patients and the healthy control signed the informed consent form.

\section{Expansion of erythroid progenitor cells isolated from whole blood}

Blood samples were collected in a vacutainer tube containing EDTA (as an anticoagulant) at room temperature. Erythroid progenitor (EP) cells were then separated through a RosetteSep method (15216; 
Stem Cell Technologies), which removed unwanted cells, such as mature RBCs and platelets, while retaining the desired EP population. Due to the low concentration of EPs in whole blood, the cells were expanded and cultured in a specific culture medium containing cytokines and supplements (X-vivo medium, Lonza). EP cells were identified by flow cytometry using the transferrin receptor (CD71) and glycophorin A (GlyA) as markers labeled with GFP (Suppl Figure S2).

\section{Reprogramming erythroid progenitor cells}

Expanded EP cells were collected and nucleofected using an Epi5 Episomal iPSC Reprogramming kit (Thermo Fisher) containing an optimized mixture of five reprogramming factors (Oct-4, Sox2, Lin28, LMyc and KIf4). Transfection (electroporation) was performed with episomal vectors (virus free, nonintegrating) from a Lonza Nucleofactor kit. After transfection, cells were plated in ReproTeSR-specific culture medium for reprogramming (Stem Cell Technologies) under standard cell culture conditions $\left(37^{\circ} \mathrm{C}, 95 \% \mathrm{CO}_{2}\right.$, and $\left.5 \% \mathrm{O}_{2}\right)$.

\section{Karyotyping}

To verify whether the reprogramming process preserved chromosomal integrity, a karyotype analysis was carried out at the Genetic Department of UNIFESP. Cells were treated with $100 \mu \mathrm{l}$ of colchicine $(0.08 \mu \mathrm{g} / \mathrm{ml})$ in $5 \mathrm{ml}$ of iPS cell culture medium to induce cell cycle arrest, which was followed by the addition of a $0.075 \mathrm{M}$ hypotonic $\mathrm{KCl}$ solution to induce nuclear swelling; cells were then fixed with a mixture of methanol and acetic acid (3:1). Metaphase chromosomes were harvested, and Wright staining was used for cytogenic analysis of G-bands (Suppl Figure S3).

\section{iPSC colony characterization}

EP cells from the healthy control $(\mathrm{HC})$ and the two ADPKD patients reprogrammed by episomal vectors formed typical iPSC colonies and presented a normal karyotype (Figure S3). The iPSC colony characteristics were compared with those of an established control (H9 embryo cells) (Supp Figure S4). As the iPSCs presented typical morphology, the pluripotency markers were identified by immunofluorescence. Cells were fixed with $4 \%$ paraformaldehyde for $30 \mathrm{~min}$ at room temperature. After fixing, samples were washed with PBS three times, blocked in 5\% PSA and 0.3\% Triton-X-100/DPBS, and incubated overnight with primary antibodies (Cell Signaling, Ma, EUA), namely, OCT4 (1:400 dilution) and NANOG (1:400 dilution), as a test of pluripotency. On the next day, a new slide was prepared, washed in PBS and incubated with an Alexa Fluor secondary antibody (1:200 dilution, Cell Signaling), and DAPI (1:1000, Invitrogen, MA, USA) was added for nuclear identification.

\section{Capacity of iPSCs to differentiate into all three germ layers}

iPSCs were grown in 6-well plates coated with Matrigel (1:10, BD Biosciences, NY, EUA) in mTeSR1 culture medium (Stem Cell Technologies, BC, Canada). To prevent the iPSCs from differentiating, the colonies were passaged manually by mechanical fragmentation with a pipet tip under a microscope, or they were dissociated by enzymatic digestion by Gentle Cell (Life Technologies) (Supp Figure S5). Using these methods, iPSCs were maintained for 22 serial passages to ensure that cells properly incorporated the 
reprogramming factors. The iPSCs were then stimulated to differentiate into the three germ layers using a STEMdiff Trilineage Differentiation kit (Stem Cell Technologies). After five days, cells were harvested for analysis of lineage-specific markers of mesoderm and endoderm, and after seven days they were analyzed for ectoderm, according to the STEMdiff Trilineage Differentiation protocol (Supp Figure S6).

\section{Molecular characterization of all three germ lines by qRT- PCR}

Total RNA from cultures of the three germ layers was extracted using a GE RNA Spin Mini kit (GE Healthcare, USA), and it was converted to cDNA using a High Capacity Reverse Transcription Kit (Applied Biosystems, USA) according to the manufacturer's instructions. Quantitative real-time PCR (qRT-PCR) was carried out using a SYBR Green PCR Kit (Applied Biosystems, USA) according to the manufacturer's protocol. mRNA expression levels were calculated using the $2^{\Delta \Delta} \mathrm{Ct}$ method. Hydroxymethylbilane synthase (HMBS) and glyceraldehyde 3-phosphate dehydrogenase (GAPDH) were used as internal controls, and the expression of OCT4 was used as a quantitative control. The expression of SOX17 was used to identify differentiation into endoderm, CXCR4 used to identify differentiation into mesoderm, and PAX6 used to identify differentiation into ectoderm (Supp Figure S7). All primer sequences are specified in Table S1, and the efficiency curve was evaluated for each primer pair.

\section{Immunofluorescence for the different germ layers}

iPSCs were induced to differentiate on coverslips in six-well plates. After differentiation, the coverslips were removed from the original wells and transferred to a new plate, where the cells were fixed with $4 \%$ paraformaldehyde and then washed with PBS, as described above. Primary antibodies against OCT4 (1:400; Cell Signaling), CXCR4 (1;100; Cell Signaling), PAX6 (1:100; Thermo Fisher), and SOX17 (1:100; R\&D Systems) were used. Fluorescence was assessed using an Alexa Fluor secondary antibody (1:200; Cell Signaling).

\section{Kidney organoid differentiation from iPSCs}

Renal organoids were generated following the protocol described by Freedman et $\mathrm{al}^{7}$ with some adaptations. Briefly, 50,000 iPSCs from the HC and two ADPKD patients were used to produce suspensions of single cells with Accutase detachment solution (Stem Cell Technologies). Single cells were supplemented with $10 \mu \mathrm{M}$ Rho-kinase inhibitor (Y27632, Stem Cell Technologies or Stemgent). After $16 \mathrm{~h}$, the culture medium was replaced with $1 \mathrm{~mL}$ of mTeSR $1+2.5 \%$ Matrigel $+10 \mu \mathrm{M}$ Y27632. After $20 \mathrm{~h}$, the medium was replaced with $1 \mathrm{~mL}$ of mTeSR 1 only; $14 \mathrm{~h}$ later, the culture medium was replaced, and cells were induced with $6 \mu \mathrm{M}$ CHIR99021 (AT104; Stem Cell or Stemgent GSK-3b inhibitor/Wnt pathway agonist) + Advanced RPMI (Thermo Fisher) + Glutamax (Life Technologies) + $10 \mu \mathrm{g} / \mathrm{ml}$ antibiotic (Ampicillin; 1:1000; Gibco). Finally, $36 \mathrm{~h}$ later, the culture medium was replaced with DRB medium containing Advanced RPMI (Thermo Fischer) + Glutamax (Thermo Fischer) + B27 Supplement (17504044; Life Technologies) + antibiotic. The DRB medium was changed every three days for 28 days. This period (28 days) was enough for the organoids to be visible by microscopy. 


\section{Molecular characterization of organoids by RT-PCR}

Semiquantitative gene expression was evaluated by RT-PCR during the embryogenic processes with total RNA being purified from the cell structures formed at days 0 (iPSCs) and at 14,21, 28, and 35 days after differentiation induction. The expression of WT1 (developing kidney), AQP1 (proximal tube), and ECAD (cyst epithelium marker) was evaluated. GAPDH was used as an internal control. The expression of OCT4 was used as a quantitative standard.

\section{Structural characterization of organoids assessed by immunofluorescence}

The specific structure of the organoids was identified by immunofluorescence using antibodies against the following proteins: Nephrin 2 (NPHS2, 1:100 dilution; Abcam, UK) for podocytes, AQP1 (1:500 dilution; Sigma; Munich, Germany) and NHE3 (1:500 dilution; Abcam) for proximal tubules, AQP2 (1:100 dilution; Abcam) for distal tubules and ECAD for cystic structures (1:500 dilution R\&D Systems). Fluorescent images were captured using an EVO microscope from Thermo Fisher. Positive and negative controls for all antibodies are shown in supplementary Figure S8.

\section{Cyst induction}

Forskolin (10 $\mu \mathrm{M}$; Sigma), an adenyl cyclase inhibitor, was used to induce cyst formation. Forskolin was added to adherent cultures on the 28th day of differentiation, since this period was coincident with the onset of tubular protein expression.

\section{Results}

\section{Obtaining iPSCs from erythroid progenitors (EPs)}

The protocol employed to obtain kidney organoids from erythroid progenitors is summarized in Fig. 1A. EPs expansion resulted in $1 \times 10^{6}$ cells after approximately 20 days (Figure S2A), and approximately two months later, EPs were identified by their expression of the markers CD71 and GlyA. The efficiency of the separation method revealed that $38.6 \%$ of the cells were appropriately sorted, as shown by GFP (fluorescent green protein) expression; $65.1 \%$ of the cells were CD71 positive, and $70.9 \%$ were GlyA positive (Figure S2B).

After verifying that the cells were EPs, they were transfected with reprogramming factors (Oct-4, Sox2, Lin28, L-Myc and klf4) and then were maintained in a specific medium for reprogramming (ReproTeSR, Stem Cell Technologies) for approximately five days. iPSCs developed typical cellular characteristics by forming colonies with a morphology distinct from that of non-reprogrammed cells present in the same cell culture. Cells were monitored until we observed typical iPSC-like colonies that were similar to the colonies of commercial H9 iPSCs (Suppl Figure S4). iPSC colonies were also recognized by their relatively larger size, tight cell packing, well-defined homogeneous shape and regular edges. Identification was 
facilitated by the low degree of colony overgrowth from differentiated cells in feeder-free reprogramming medium (Fig. 1B). No detectable differences were found among iPSC colonies from the HC and ADPKD patients (PT1 and PT2) in terms of colony morphology and pluripotency markers, as shown in Fig. 1C.

iPSCs were further characterized by their ability to differentiate into the three germ layers. Typical images of the progression of germ layer development in cells from the HC are shown in supplementary Figure S6. Development of the three germ layers was verified by typical morphology (Fig. 2A) and by immunofluorescence labeling for differentiation markers, namely, FOXA2 for endoderm, smooth muscle actin (SMA) for mesoderm and SOX2 for ectoderm (Figs. 2B-D). Figure 2E shows the gene expression levels of specific markers of endoderm (SOX17), mesoderm (CXCR4) and ectoderm (PAX6), as detected by RT-PCR.

\section{Generation of kidney organoids from iPSCs}

Three-dimensional renal structures were generated from iPSCs derived from the $\mathrm{HC}$ and both ADPKD patients following steps that recapitulate embryonic development. Figure 3 shows the steps that are necessary to obtain the respective embryonic structures. iPSC colonies (Fig. 3a) were dissociated with Accutase (3b) to obtain single iPSCs (3c). Considering the normal embryogenic process, the colonies would be expected to differentiate into a blastula and then a gastrula, but the spontaneous differentiation of iPSCs in culture is still a challenge since cells spontaneously undergo apoptosis ${ }^{17}$. To circumvent this undesired outcome, a ROCK inhibitor of signaling (10 $\mathrm{M}$ Rho-kinase inhibitor Y27632) was added $16 \mathrm{~h}$ after the single cell stage. Inhibition of the ROCK pathway prevents cells from triggering the death pathways, allowing them to survive and adjust to proceed towards the differentiation process. The iPSCs then grew until reaching the epiblast stage, which is characterized by the presence of spheroid cavities (3d). Next, a potent GSK-3b inhibitor/Wnt pathway agonist (CHIR99021) was added, which was followed by the addition of DRB medium to induce epithelial-mesenchymal transition (3e-f) and finally mesenchyme-epithelial transition $(3 \mathrm{~g}$ ); these steps enabled epithelialization and sequential formation of pretubular aggregates and renal vesicles $(3 \mathrm{~h})$, which ultimately enabled the formation of renal tubular organoids (3i). All these in vitro embryogenesis steps were similar for cells derived from both $\mathrm{HC}$ and ADPKD patients.

The presence of tubular structures was verified by the expression of specific markers by immunofluorescence: NHE3 and AQP1 for proximal tubules (Fig. 4A) and NPHS2 for glomeruli (Fig. 4B). In contrast, AQP2 labeling was not detected (Fig. 4B), confirming that this methodology allowed only the development of the metanephric mass but not of the ureteric bud. The marker ECAD was not detected in the absence of forskolin (4B).

To verify the presence of specific markers expressed throughout the process of progenitor kidney development, all samples (HC, PT1 and PT2) were collected at five points of the whole process of differentiation: day 0 (iPSCs), days 14, 21, 28 and then at day 35 (7 days after cyst induction). The time course of gene expression revealed differentiation during the embryogenesis process, as shown in Fig. 5A-C. The pluripotency marker OCT4 was expressed by iPSCs (day 0), but its levels were reduced over 
14 days, reaching very low levels following differentiation. In contrast, the expression of the specific markers of metanephric mesenchyme (WT1) and proximal tubule (AQP1) progressively increased when the differentiation process was initiated by day 14 , reaching a plateau by day 21 . Figure $5 \mathrm{D}$ shows the expression of ECAD, a cystogenesis marker, at day 35, i.e. 7 days after cyst induction by forskolin. In the absence of forskolin, the expression of ECAD was very low, even in ADPKD patients. However, when forskolin was added (day 28), both patients exhibited increased ECAD expression; but the same pattern was not observed in $\mathrm{HC}$ organoid which remained with low levels of ECAD expression even in the presence of forskolin.

The presence of cysts formed from the organoids stimulated with forskolin was evident for ADPKD patients, as shown in Fig. 6 . The upper panel shows images of the organoids at day 28, before the addition of forskolin. (Fig. 6Aa). Figures 6Ab (panoramic view-4X) and 6Ac (20X viewing) show the organoids at day 35 in the presence of forskolin showing the presence of cyst-like structures in samples from both patients (PT1 and PT2) but not in the HC sample. The presence of cysts was also verified by immunofluorescence staining (Fig. 6B). ECAD labeling was not detected in the $\mathrm{HC}$ organoid, but it was clearly observed in the organoids from both patients. Interestingly, the presence of a cyst lumen can also be observed.

\section{Discussion}

In the present study, we described in detail a procedure to generate kidney organoids from human iPSCs obtained from the blood erythroid progenitor cells of patients with ADPKD. Many somatic cell types have been successfully reprogrammed into iPSCs, including blood cells ${ }^{8}$. Here, we opted to use blood erythroid progenitors as the starting cell type for a number of reasons, such as their genomic integrity, epigenetic memory and efficient reprogramming ${ }^{12,18,19}$. The efficiency by which erythroid progenitors had been converted into iPSCs was as much as two orders of magnitude better $(7 \%-28 \%)$ than the efficiency of differentiated blood cell types $(0.02 \%-0.60 \%)$ or fibroblasts $(0.74 \%)^{5}$. Despite the low abundance of these cells in the peripheral blood, they can easily be isolated and expanded in vitro to produce a sufficient number for reprogramming. One of the most important challenges in establishing suitable iPSCs is their genotype and phenotypic variability ${ }^{7}$, but there are some technical strategies proposed to circumvent or reduce such variability. In the present experiments, we used a non-integrating episomal system for reprogramming that does not require genome integration for the reprogramming genes to be expressed ${ }^{11}$, allowing less immunogenicity and rendering more genetically stable cells ${ }^{20,21}$. Moreover, all samples were derived from the same source, which helps minimize epigenetic variability ${ }^{13}$, assuming that differentiation of the starting cell might influence the efficiency of the end results. Analysis of the pluripotency markers and the ability of iPSCs to differentiate into all three germ layers were compared side-by-side, and they were also compared to the $\mathrm{H} 9$ embryonic cell line, which was used as a positive control. All these comparisons showed no significant differences between ADPKD patients, HCs and H9 cells. 
Several protocols have been developed for inducing the differentiation of iPSCs into kidney organoids ${ }^{22,}$ 23. Based on protocols described by Cruz et al ${ }^{7}$, we obtained structures with the characteristics of proximal tubules, including the expression of NHE3 and AQP1. In addition, the organoids expressed NPHS2, indicating the presence of podocyte-like cells. Conversely, AQP2 was not expressed in these tubular organoids. This finding is in agreement with previous studies pointing to several limitations in developing structures derived from the ureteric bud (UB) in vitro, with the development of UB requiring specific protocols, since the timing window for the addition of the specific inductor is very limited $7,24,25$.

In the process of generating kidney organoids from iPSCs, all of renal embryogenesis is recapitulated in vitro, initially involving the formation of cavitated spheroids from single cells ${ }^{15}$. Indeed, we found that undifferentiated iPSCs from both ADPKD patients and healthy control formed epiblast-like spheroid structures that were morphologically similar. Although DNA sequencing of the PKD1 and PKD2 genes was not performed in the present study, the current findings indicate that the type of mutation carried by the patients had no influence on typical epiblast morphogenesis. Further studies are still needed to more properly evaluate the expression of pivotal molecules involved in this phase of embryonic development.

According to the protocol developed by Freedman et al ${ }^{15}$, we observed that spheroids did differentiate into tubular organoids 26 days after induction of differentiation, and the timing was similar for both ADPKD patients and HC (Fig. 3). Nevertheless, we noticed some variability regarding the timetable of expression and the levels of differentiation markers. On the other hand, the morphology of the tubular structures was similar and comparable among samples from the ADPKD patients and HC. In contrast, using iPSCs originating from renal biopsies of ADPKD patients, Freedman's group found a higher variability in the ability of these cells to form organoids ${ }^{7}$. In addition to the huge diversity of mutations inherent to this disease, these differences in the efficiency of obtaining organoids may also be explained by the heterogeneous cell types present in biopsy samples, in contrast to the erythroid progenitors, which constitute a more homogeneous cell population. Taken together, these results indicate that despite the variability and differences in the efficiency of different iPSC clones to form kidney organoids, obtaining the latter from ADPKD patients may constitute a unique model for studying the multiple forms of phenotypical manifestations of this disease. Moreover, it is important to note that if there is a considerable variability of mutations in the ADPKD-affected population, the patient-specific iPSCs, being totally immune compatible, they might be used for individual regenerative therapies in the future, in addition to allowing the development of an in vitro model to verify whether the very early phenotypical manifestations are also as diverse as the clinical presentation.

Unstimulated organoids derived from HC or ADPKD patients did not spontaneously form cysts. The typical morphology of cysts and the expression of cystogenesis markers were not detectable in any sample, even extending the organoid differentiation in culture until completing 35 days. It has been demonstrated that cell proliferation alone does not produce cysts ${ }^{26}$. Moreover, a third independent event (known as third-hit) is required to accelerate cyst formation and growth in vivo ${ }^{25}$. Conversely, cyst formation induced by forskolin did occur in the organoids from ADPKD patients but not in those from HC. 
Of note, although present, cysts were observed at a low percentage in the ADPKD samples even in the presence of forskolin, as has also been observed by Freedman et $\mathrm{al}^{15}$ in organoids formed from the CRISPR/Cas9 model of ADPKD disease. However, when adherent organoids were transferred to a 3D suspension in their model, cystogenesis increased $10-\mathrm{fold}^{7}$, indicating that the microenvironment is directly implicated in cystogenesis. The low percentage of cysts in the ADPKD organoids in the current study could be accounted for by the use of adherent organoids.

Aside from the role of the microenvironment in enabling efficient cyst formation in vitro, the experimental protocol employed in the present study formed proximal tubule organoids but not more distal portions of the nephron ${ }^{25}$. Notably, it is well established that in vivo, the proximal tubule does not represent the main site of cyst formation, corresponding to approximately $1.8 \% 27$, whereas the distal nephron (particularly the collecting duct) is the main site of cyst origin.

\section{Conclusion}

In conclusion, the present findings showed that it is feasible to generate organoids with three-dimensional structures similar to the kidney progenitor architecture using iPSCs reprogrammed from erythroid progenitors that were easily isolated from ADPKD patients and that these organoids are able to form

cysts under forskolin stimulation. This promising methodology unveils a method for increasing understanding of the very early genetic, phenotypical and physiological steps in cyst formation in the ADPKD setting.

\section{Declarations}

\section{Author Contributions}

R.F. and M.A.B. designed the study; R.F., F.H.L., A.C.A. carried out experiments; R.F., F.H.L., E.M., M.C.S. and M.A.B analyzed the data; J.L.N. and I.P.H. patients clinical control, selection and interview; R.F. and M.A.B. made the figures; R.F., I.P.H., M.C.S and M.A.B. drafted and revised the paper. All authors approved the final version of the manuscript.

\section{Funding Section}

This work was supported by funding from CNPq, CAPES, FAPESP (grants 2015/23345-9 to MAB and No. 2016/05311-2 to MCS), and Fundação Oswaldo Ramos.

\section{Acknowledgements}

Not applicable. 


\section{Ethics approval and consent to participate}

The study was reviewed and approved by the Ethics Advisory Committee of the São Paulo University (Nr.1199.-0079-10/2018).

\section{Availability of data and materials}

All data generated or analyzed during this study are included in this article.

\section{Competing interests}

The authors declare that they have no competing interests.

\section{Consent for publication}

Authors have no conflict of interest.

\section{Abbreviations}

iPSCs: induced pluripotent stem cell; ADPKD:autosomal dominant polycystic kidney disease; EP:erythroid progenitors; HC health control; PC-1:polycystic1; PC2:polycystic2; PDK:polycystic disease; GLYA glycophorin A; qRT-PCR: quantitative real time PCR; HMBS: (hydroxymethylbilane synthase; GAPDH: gliceroldehyde 3-phosphatase dehydrogenase; NPHS2: Nephrin2; AQP1:aquaporin1; AQP2:aquaporin2; $\mathrm{NH} 3: \mathrm{NA}+/ \mathrm{H}+$ exchanger isoform3; WT1:Wilms tumor; ECAD:E-cadherin; UB:ureteric bud; MM:metanephric mass; DNA:deoxyribonucleic acid; CRISPR/cas9:clustered regularly interspace short palindromic repeats; Oct4: octamer-binding transcription factor 4; SMA:smooth muscle actin;

\section{References}

1. Chebib FT, Torres VE. Autosomal Dominant Polycystic Kidney Disease: Core Curriculum 2016. Am J Kidney Dis. 2016;67::792-810.

2. Watnick TJ, Torres VE, Gandolph MA, Qian F, Onuchic LF, Klinger KW, Landes G, Germino GG. Somatic mutation in individual liver cysts supports a two-hit model of cystogenesis in autosomal dominant polycystic kidney disease.1998, Mol Cell, 2: 247-251.

3. Pei Y, Watnick T, He N, Wang K, Liang Y, Parfrey P, Germino G, St George-Hyslop P. Somatic PKD2 mutations in individual kidney and liver cysts support a "two-hit" model of cystogenesis in type 2 autosomal dominant polycystic kidney disease. J Am Soc Nephrol. 1999;10::1524-9.

4. Piontek K, Menezes LF, Garcia-Gonzalez MA, Huso DL, Germino GG. A critical developmental switch defines the kinetics of kidney cyst formation after loss of Pkd1. Nat Med. 2007;13::1490-5. 
5. Lantinga-van Leeuwen IS, Leonhard WN, van der Wal A, Breuning MH, de Heer E, Peters DJ. Kidneyspecific inactivation of the $\mathrm{Pkd} 1$ gene induces rapid cyst formation in developing kidneys and a slow onset of disease in adult mice. Hum Mol Genet. 2007;16::3188-96.

6. Takakura A, Contrino L, Zhou X, Bonventre JV, Sun Y, Humphreys BD, Zhou J. Renal injury is a third hit promoting rapid development of adult polycystic kidney disease. Hum Mol Genet. 2009;18::252331.

7. Cruz NM, Song X, Czerniecki SM, Gulieva RE, Churchill AJ, Kim YK, Winston K, Tran LM, Diaz MA, Fu H, Finn LS, Pei Y, Himmelfarb J, Freedman BS. Organoid cystogenesis reveals a critical role of microenvironment in human polycystic kidney disease. Nat Mater. 2017;16::1112-9.

8. Takahashi K, Okita K, Nakagawa M, Yamanaka S. Induction of pluripotent stem cells from fibroblast cultures. Nat Protoc. 2007;2::3081-9.

9. Freedman BS, Lam AQ, Sundsbak JL, latrino R, Su X, Koon SJ, Wu M, Daheron L, Harris PC, Zhou J, Bonventre JV. Reduced ciliary polycystin-2 in induced pluripotent stem cells from polycystic kidney disease patients with PKD1 mutations. J Am Soc Nephrol. 2013;24::1571-86.

10. 9.1-4A.9.10

Chen HY, Tan HK, Loh YH: Derivation of Transgene-Free Induced Pluripotent Stem Cells from a Single Drop of Blood. Curr Protoc Stem Cell Biol, 2016, 38: 4A.9.1-4A.9.10. DOI: 10.1002/cpsc.12.

11. Yu J, Hu K, Smuga-Otto K, Tian S, Stewart R, Slukvin II, Thomson JA. Human induced pluripotent stem cells free of vector and transgene sequences. Science. 2009;324::797-801.

12. Eminli S, Foudi A, Stadtfeld M, Maherali N, Ahfeldt T, Mostoslavsky G, Hock H, Hochedlinger K. Differentiation stage determines potential of hematopoietic cells for reprogramming into induced pluripotent stem cells. Nat Genet. 2009;41::968-76.

13. Chou BK, Mali P, Huang X, Ye Z, Dowey SN, Resar LM, Zou C, Zhang YA, Tong J, Cheng L. Efficient human iPS cell derivation by a non-integrating plasmid from blood cells with unique epigenetic and gene expression signatures. Cell Res. 2011;21::518-29.

14. Allison SJ. Tubuloids for modelling kidney disease. Nat Rev Nephrol. 2019;15:319.

15. Freedman BS, Brooks CR, Lam AQ, Fu H, Morizane R, Agrawal V, Saad AF, Li MK, Hughes MR, Werff RV, Peters DT, Lu J, Baccei A, Siedlecki AM, Valerius MT, Musunuru K, McNagny KM, Steinman TI, Zhou J, Lerou PH, Bonventre, JV: Modelling kidney disease with CRISPR-mutant kidney organoids derived from human pluripotent epiblast spheroids. Nat Commun, 2015, 6: 8715.

16. Pei Y, Obaji J, Dupuis A, Paterson AD, Magistroni R, Dicks E, Parfrey P, Cramer B, Coto E, Torra R, San Millan JL, Gibson R, Breuning M, Peters D, Ravine D. Unified criteria for ultrasonographic diagnosis of ADPKD. J Am Soc Nephrol. 2009;20::205-12.

17. Watanabe K, Ueno M, Kamiya D, Nishiyama A, Matsumura M, Wataya T, Takahashi JB, Nishikawa S, Muguruma K, Sasai Y. A ROCK inhibitor permits survival of dissociated human embryonic stem cells. Nat Biotechnol. 2007;25::681-6.

18. Hochedlinger K, Jaenisch R. Nuclear reprogramming and pluripotency. Nature. 2006;441::1061-7. 
19. Maherali N, Ahfeldt T, Rigamonti A, Utikal J, Cowan C, Hochedlinger K. A high-efficiency system for the generation and study of human induced pluripotent stem cells. Cell Stem Cell. 2008;3::340-5.

20. Briggs JA, Sun J, Shepherd J, Ovchinnikov DA, Chung TL, Nayler SP, Kao LP, Morrow CA, Thakar NY, Soo SY, Peura T, Grimmond S, Wolvetang EJ. Integration-free induced pluripotent stem cells model genetic and neural developmental features of down syndrome etiology. Stem Cells. 2013;31::46778.

21. Rao MS, Malik N. Assessing iPSC reprogramming methods for their suitability in translational medicine. J Cell Biochem. 2012;113::3061-8.

22. Little MH, Combes AN. Kidney organoids: accurate models or fortunate accidents. Genes Dev. 2019;33::1319-45.

23. Schutgens F, Verhaar MC, Rookmaaker MB. Pluripotent stem cell-derived kidney organoids: An in vivo-like in vitro technology. Eur J Pharmacol. 2016;790:12-20.

24. Nishinakamura R. Human kidney organoids: progress and remaining challenges. Nat Rev Nephrol. 2019;15::613-24.

25. Taguchi A, Nishinakamura R. Higher-Order Kidney Organogenesis from Pluripotent Stem Cells. Cell Stem Cell. 2017;21::730-46.e736.

26. Yamaguchi T, Pelling JC, Ramaswamy NT, Eppler JW, Wallace DP, Nagao S, Rome LA, Sullivan LP, Grantham JJ. cAMP stimulates the in vitro proliferation of renal cyst epithelial cells by activating the extracellular signal-regulated kinase pathway. Kidney Int. 2000;57::1460-71.

27. Grantham JJ, Geiser JL, Evan AP. Cyst formation and growth in autosomal dominant polycystic kidney disease. Kidney Int. 1987;31::1145-52.

\section{Figures}


A

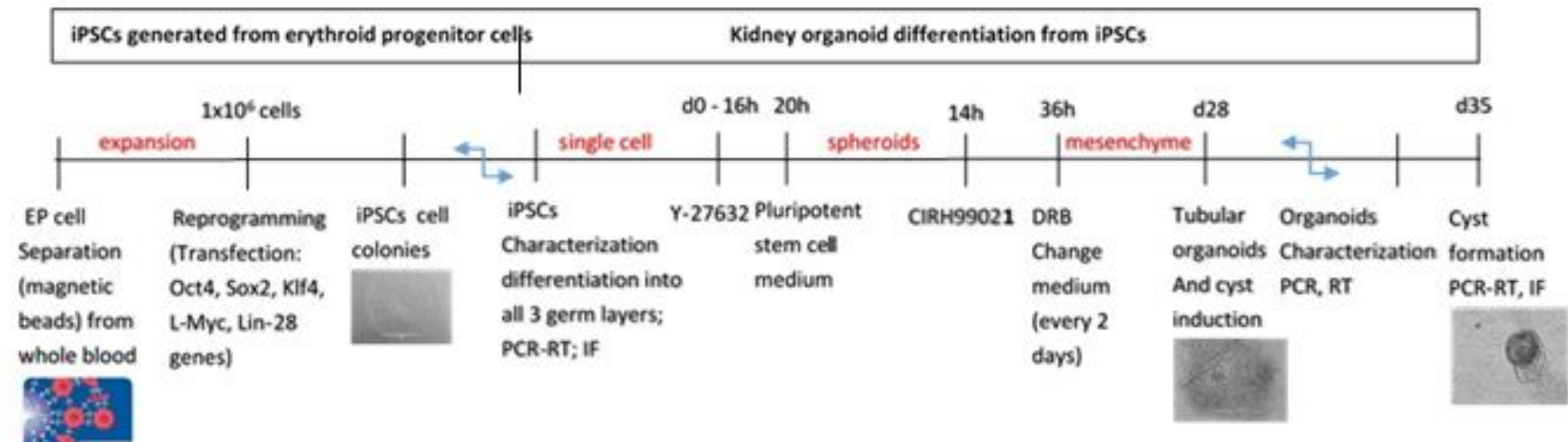

B

HC

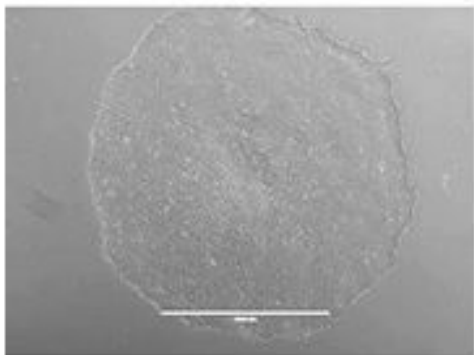

C

DAPI

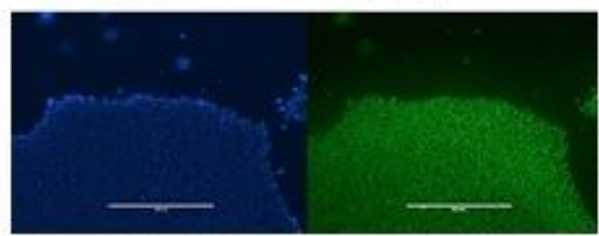

PT1

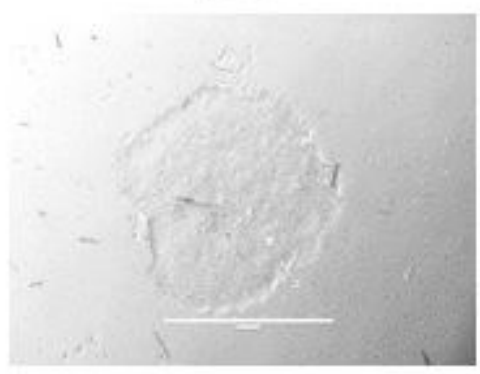

DAPI

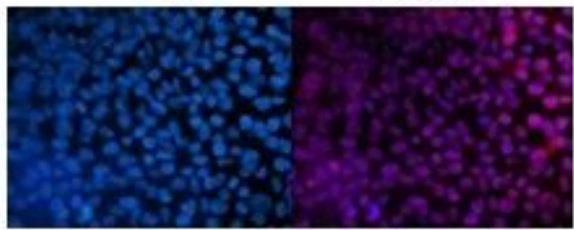

PT2

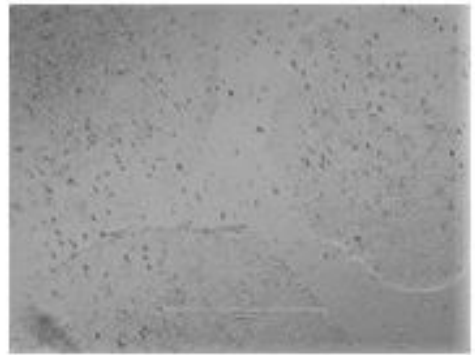

DAPI

Nanog

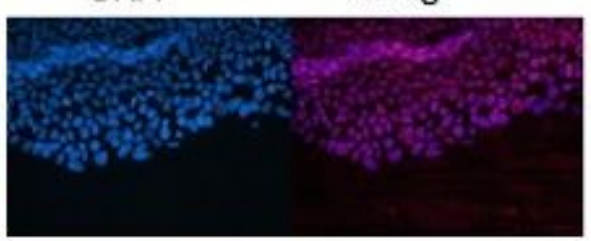

\section{Figure 1}

Generation and characterization of iPSCs. iPSCs were reprogrammed from expanded erythroid progenitor (EP) cells from a healthy control (HC) and two different ADPKD patients (PT1 and PT2). A. Schematic stepwise generation of kidney organoids. B. Morphology of iPSC colonies. C. Characterization of iPSC pluripotency observed under an EVOS fl inverted digital fluorescence microscope (scale: 1,000 $\mu \mathrm{m}$ ). Two antibodies were used for this purpose: OCT4 labeling was used to characterize pluripotency of the HC colony, and Nanog was used as a pluripotency marker for PT1 and PT2. Cell nuclei were labeled with DAPI. 


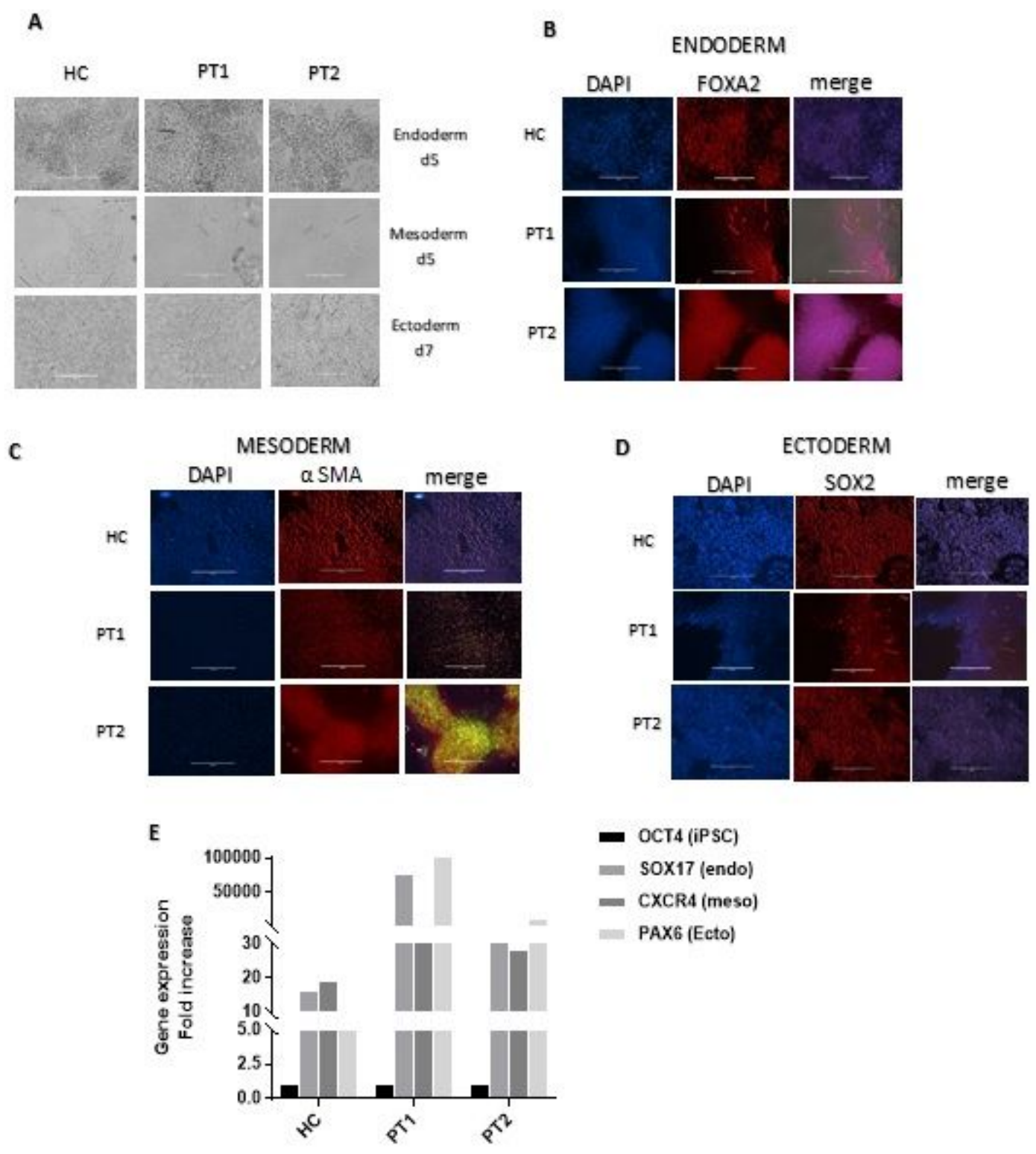

Figure 2

Characterization of the three germ layers. Healthy control (HC) and ADPKD1 patients (PT1 and PT2). A. Typical morphology of the three germ layers. Germ layers were characterized by immunofluorescence staining using specific antibodies. B. Endoderm (FOXA2), C. Mesoderm (a SMA) and D. Ectoderm (SOX2). Cell nuclei were stained with DAPI. The last columns show merged images. E. Gene expression of OCT4 indicates pluripotency of iPSCs, SOX17 was a marker for endoderm, CXCR4 was a marker for mesoderm and PAX6 was a marker for ectoderm. OCT4 expression by iPSCs was used as an internal quantitative control. OCT4 expression was undetectable in cells of the three germ layers. 

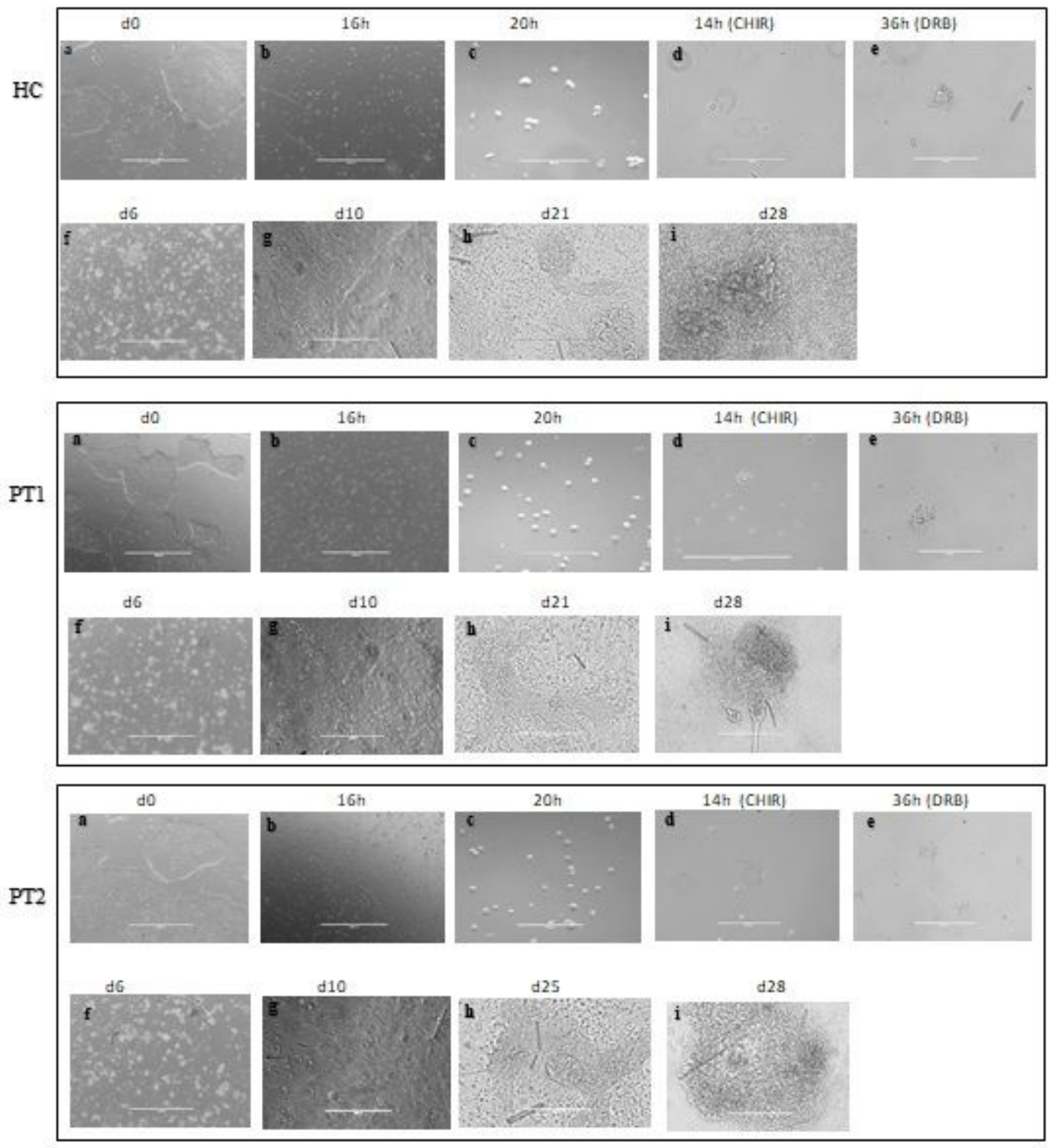

\section{Figure 3}

Generation of tubular organoids. Phase contrast images of the generation of epithelial tubular kidney organoids at 28 days from iPSCs generated from the HC, PT1 and PT2 groups. In the first $36 \mathrm{~h}$, the iPSCs were maintained in 3D culture; then, they were dissociated, transformed into single cells and treated with Y-27632, which allowed cellular reorganization and survival, producing epiblast spheroids (d). Then, CHIR99201 and DRB were added, activating the Wnt/ $\beta$-catenin pathway and allowing differentiation into tubular organoids (i). The morphology among spheroids from ADPKD patients and a HC donor is similar. 


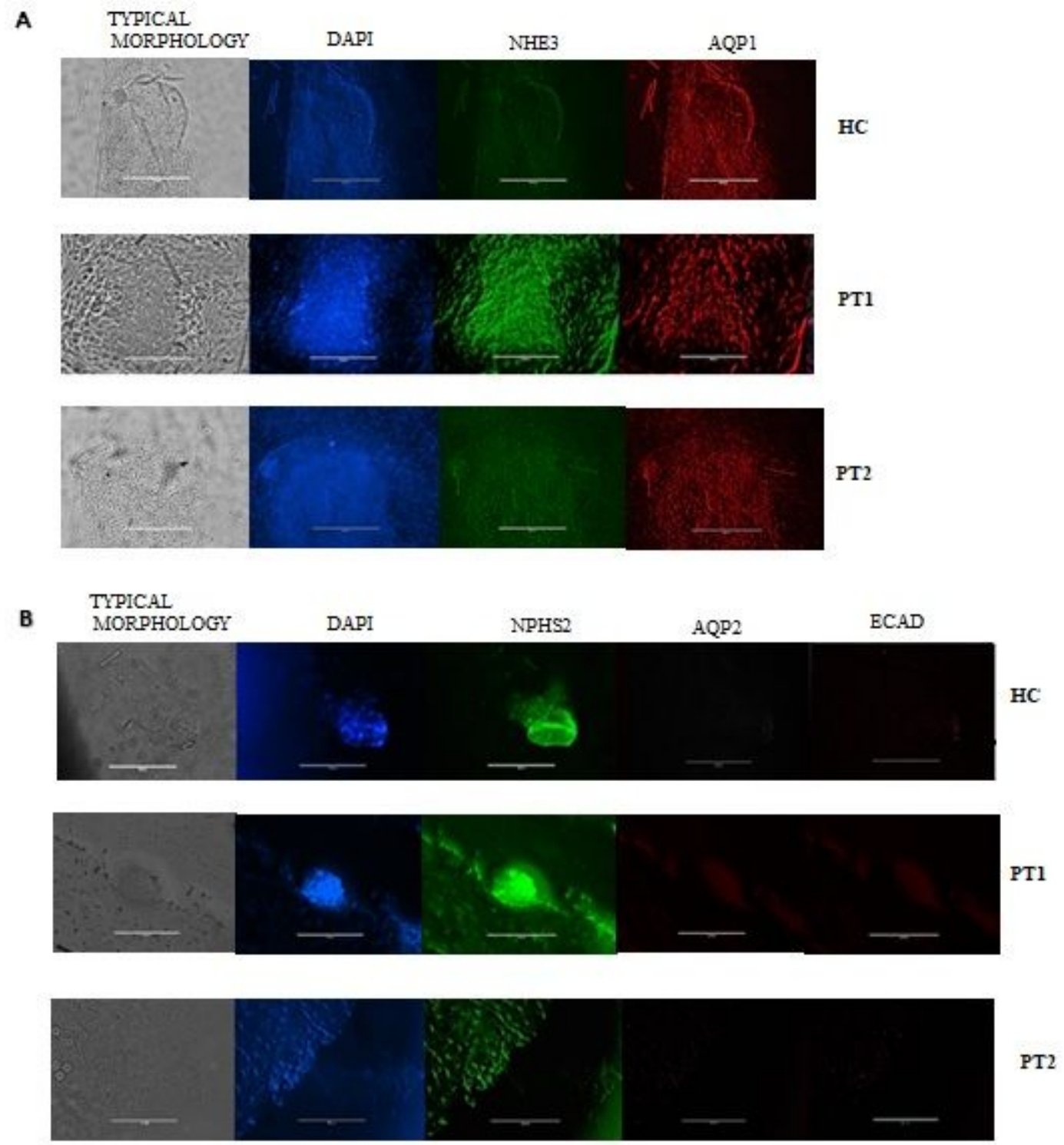

\section{Figure 4}

Kidney organoids. Representative immunostaining of epithelial tubular kidney organoids at 28 days for HC, PT1 and PT2 patients. Typical morphology of organoid structures (first columns). Cell nuclei were stained with DAPI (second columns A-B). A, each structure was characterized by immunofluorescence staining using specific antibodies against NHE3 (Abcam 1:500) and AQP1 (Sigma 1:500). B, NPHS2 (Abcam 1:100) was detected. AQP2 (Sigma 1:100) was not detectable, proving that only the metanephric mass of the structures was performed. ECAD (Sigma 1:100) was not detectable in the absence of forskolin. A-B: Images taken at 20X; scale bar $1000 \mu \mathrm{m}$. 
A

OCT4
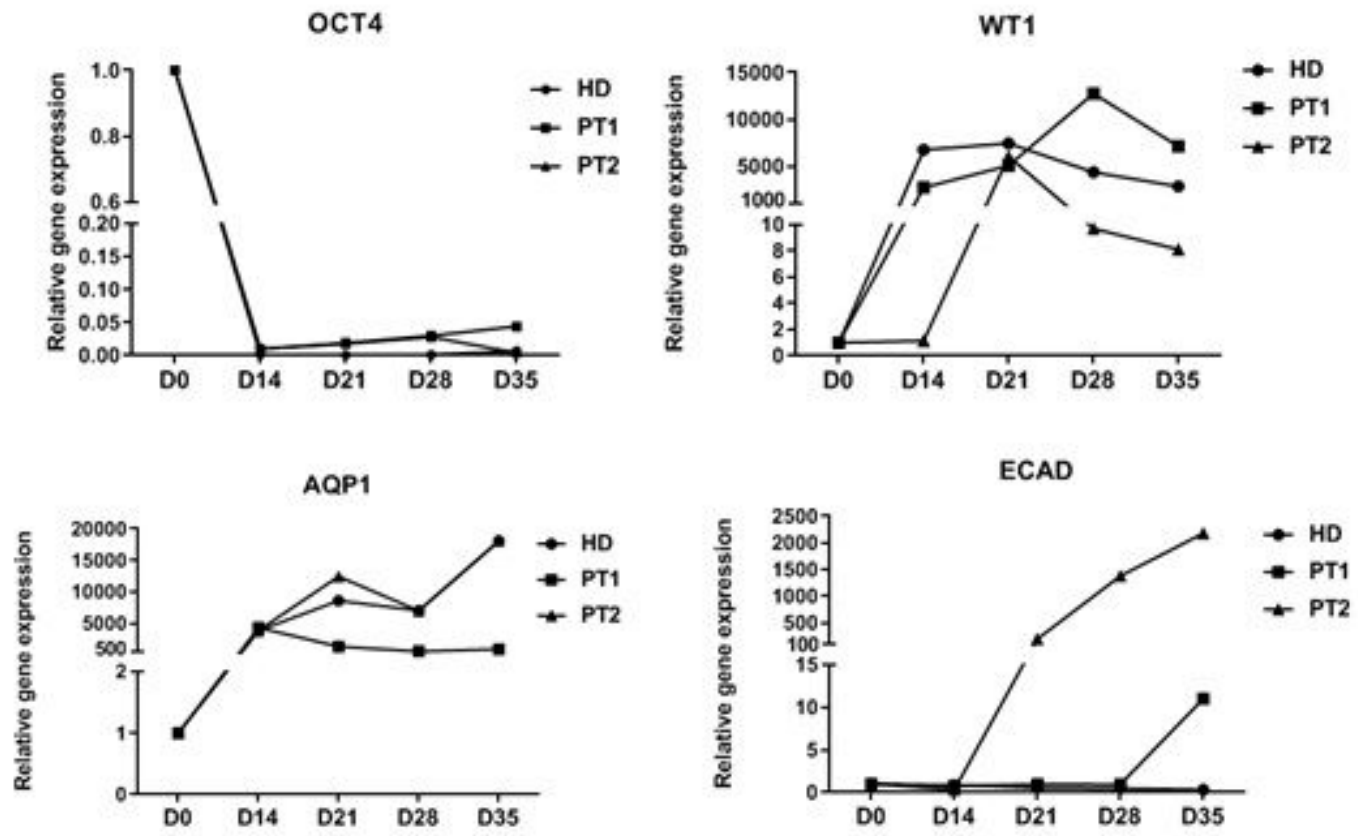

B

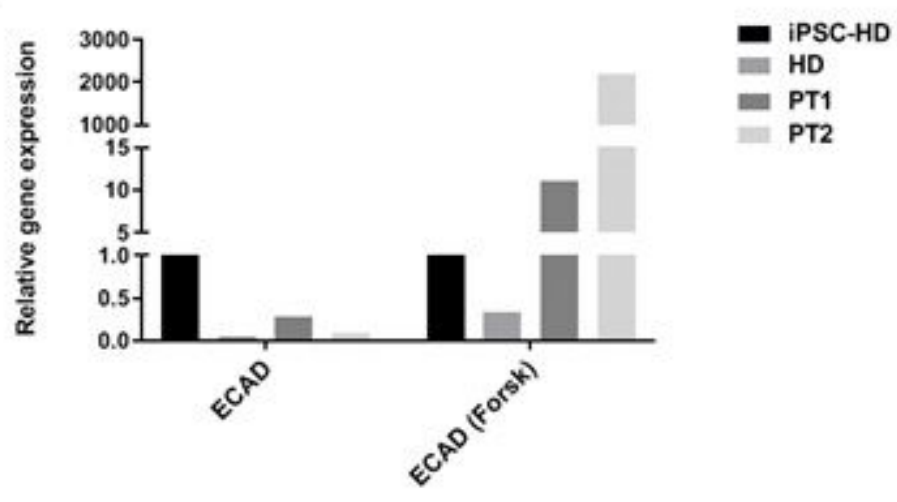

Figure 5

Relative gene expression levels of renal structures. A-C. Sequential expression of pluripotency and organogenesis markers. Day 0 (D0) refers to iPSCs and OCT4 expression in D0 was used as a quantitative standard for each sample. In this set of experiments, forskolin was added at day 28 . D. Expression levels ECAD in the organoids at day 35 in the presence and absence of forskolin (added at day 28). GAPDH was used as an internal control and OCT4 expression by iPSCs from healthy donor (HD) was used as the quantitative standard. WT1 and AQP1 were used as markers of renal structures, and ECAD as the cyst marker. 
A

HC
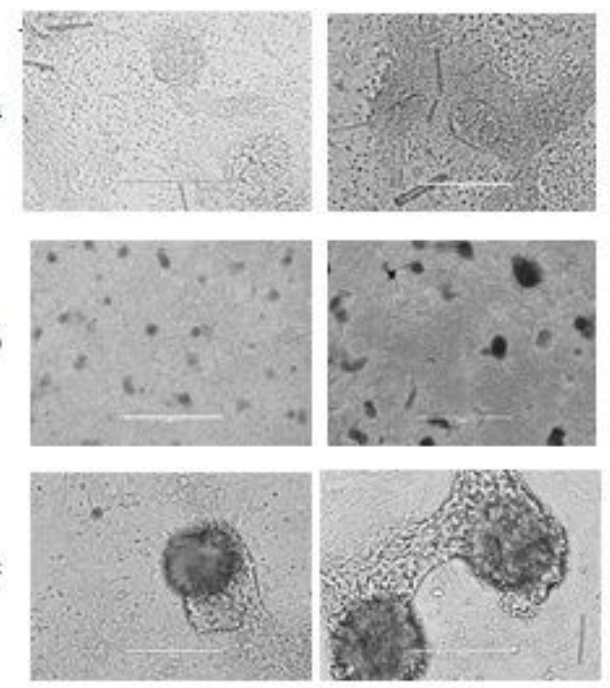

B
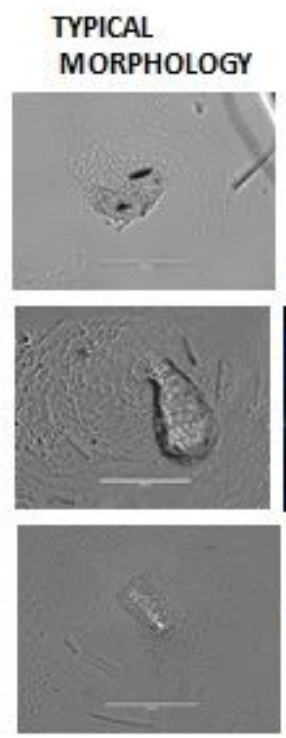

PT1
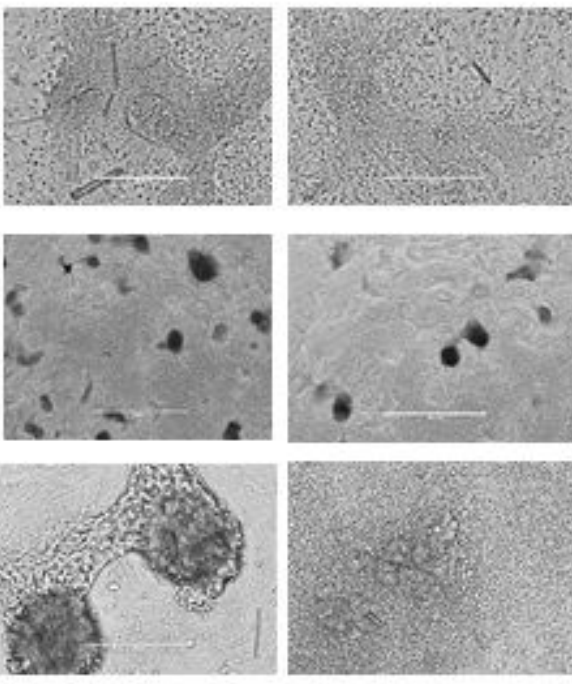

d28

$20 \mathrm{X}$

d35, Forskolin

$4 \mathrm{X}$

d35, Forskolin

$20 \mathrm{X}$

\section{Figure 6}

Organoid and cyst formation. Representative images of organoids after cyst induction with forskolin for HC, PT1 and PT2. Typical morphology of organoids on day 28 without forskolin induction (A-a). Images of several organoids on day 35 in culture upon forskolin induction 4X (A-b). Typical morphology on day 35 upon forskolin induction showing cyst formation, 20X (A-c). B Representative immunostaining of ECAD markers showing the typical morphology of kidney organoids. Cell nuclei were stained with DAPI (second columns). ECAD was not detected in the HC (R\&D Systems 1:500), but it was detected in PT1 and PT2. A-B: 20X, scale bar $1000 \mu \mathrm{m}$.

\section{Supplementary Files}

This is a list of supplementary files associated with this preprint. Click to download. 
- SUPPLEMENTS.docx

Page 20/20 